\title{
Sharbat Ahmad Shahi: A Potent Unani Formulation for Mood Disorders
}

\author{
Afshan Khan ${ }^{1}$, Aisha Siddiqui ${ }^{2 *}$, M.A Jafri ${ }^{3}$, Mohd Asif ${ }^{4}$ \\ ${ }^{1,2,3,4}$ Dept. of Ilmul Advia, School of Unani Medical Education and Research, Jamia Hamdard, New Delhi. India. \\ $2^{*}$ Corresponding Author: Aisha Siddiqui, afshankhan2490@gmail.com
}

Available online at: www.isroset.org

Received: 10/Dec/2018, Accepted: 22/Dec/2018, Online: 31/Dec/2018

\begin{abstract}
Mood disorders such as depression are the most ubiquitous disease amongst psychiatric disorders. According to the estimations of the World Health Organisation, depression will be the second leading cause of disability in 2020. Psychiatric disorders are the foremost problems in the world since relic. In Unani system of medicine, there is no any direct description of depression but it is described in detail under the heading of "Amraze nafsaniya". This system has a gem of valuable single and compound formulations for psychiatric disorders as there are several mufrad (Single) drugs used to treat psychiatric disorders such as Aftimoon (Cuscuta reflexa), Badranjboya (Nepeta hindostana), Nilofar (Nymphaea alba), Gule surkh (Rosa damascena), Bisfaij (Polypodium vulgare) etc. Sharbat Ahmad Shahi is one of the polyherbal Unani preparations and it has been conventionally used in Malankholia (Melancholia), Insanity, Mania and for saudavi amraaz. It has been reported for its antidepressant potential in recent years.
\end{abstract}

Key Words: Antidepressant, Mood disorders, Sharbat Ahmad Shahi, Unani medicine.

\section{INTRODUCTION}

Mood disorders such as depression are the most prevalent diseases amongst psychiatric disorders and a leading cause for disability worldwide. Depression, officially termed major depressive disorder (MDD) ranks among the most rampant diseases worldwide. Depression is diagnosed by the method, mentioned in the Diagnostic Manual of Mental Disorders (DSM-IV), which is characterized by the following symptoms: (1) depressed or irritable mood, (2) decreased interest or loss of pleasure, (3) weight gain or loss, (4) insomnia or hypersomnia, (5) psychomotor retardation or agitation, (6) fatigue or loss of energy, (7) feelings of worthlessness or inappropriate guilt, (8) diminished ability to think or concentrate, (9) recurrent thoughts of death and suicide. These symptoms must manifest daily for at least 2 weeks. The World Health Organization anticipated that depression will be the second leading cause of infirmity in 2020 [1]. Recent epidemiological studies specify that severe forms of depression affect $2-5 \%$ of the population worldwide, and up to $20 \%$ are influenced by milder forms of the disease [2]. Major depression is a serious disorder of massive sociological and clinical relevance. In conventional system of medicine, treatment outcome of depression is suboptimal. The use of currently available antidepressants is confined by their side effects, slow response, and inadequate treatment efficacy. Full remission is difficult to be achieved. Depression, as such is not mentioned in Unani classical texts as an individual disease entity rather it is mentioned as a symptom or group of symptoms of Malankholia (Melancholia) in which the mental functions of the individual are deranged leading to constant grief, fear and dubious aggression. The patient find himself/herself deserted and occupied by loneliness and some patients experience delusion and hallucination also [3]. In Unani medicine, psychiatric disorders are dealt in detail under the heading of "Amraze nafsaniya" where it is described by various symptoms of psychic faculty and their distortion due to the involvement of vitiated humours. Unani System of medicine believes in holistic accession to the prevention and treatment of diseases. It covers physical, mental and spiritual dimensions of an individual's health. Several drugs have been reported to have beneficial effects in psychiatric (mental) disorders. There are several mufrad (Single) drugs used to treat psychiatric disorders such as Aftimoon (Cuscuta reflexa), Badranjboya (Nepeta hindostana), Nilofar (Nymphaea alba), Gule surkh (Rosa damascena), Bisfaij (Polypodium vulgare) etc and many murakkab (compound formulations) such as Khamira Gaozaban, Sharbat Aftimoon, Sharbat Ahmad Shahi and Itrifal Kishneezi etc have been reported to have beneficial effect in psychiatric (mental) disorders. Sharbat Ahmed Shahi (SAS) is one of them. It is a compound formulation usually prescribed for the patients of depression, insanity, melancholia, mania and for black bile disorders (Saudawi amraz) in Unani system of medicine for a long time due to its minimum side effects as compared to its counter synthetic antidepressants [4][5][6].

\section{SHARBAT:}

The word Sharbat is derived from Persian "Sharbat", and sherbet is from Turkish "Serbet", both of which in turn come 
from Arabic "Sharba" a drink, from "Shariba" to drink. Sharbat is an important invention of a renowned Unani physician. It was invented by 'Hakeem Fesaghoras'. It is a sweet viscous liquid or those medicinal preparations which are made either by preparing the decoction from the plant, animal and mineral origin drugs or by taking juice of the fruits from different plants and mixed with Sugar and boiled to the required consistency (Qiwam) i.e one tar [6][177][178].

\section{METHOD OF PREPARATION OF SHARBAT:}

Dry herbal drugs are soaked overnight in water, amounting to 8 or 10 times the weight of the drugs. Next morning, they INGREDIENTS OF SHARBAT AHMAD SHAHI [6] [22] are boiled till one third water is left, allowed to cool, rubbed with hands, and filtered through a piece of fine cloth. Then, two or three times of sugar are added, and the mixture is boiled on a low fire to obtain the desired consistency of Sharbat [177].

\section{SHARBAT AHMAD SHAHI:}

Sharbat Ahmed Shahi (SAS) is one of the polyherbal compound formulations. It has been traditionally used for the treatment of Malikholia (melancholia), Mania, psychoneurosis and palpitation. It is beneficial in cerebral insufficiency, also used in chronic constipation [4][6].

\begin{tabular}{|c|c|c|c|c|}
\hline S.No & Unani Name & Botanical Name & Parts Used & Quantity \\
\hline $\mathbf{1}$ & Aftimoon & Cuscuta reflexa Roxb. & Dried leaves & $24 \mathrm{~g}$ \\
\hline $\mathbf{2}$ & Badranjboya & Nepeta hindostana Roth. & Leaves & $225 \mathrm{~g}$ \\
\hline $\mathbf{3}$ & Berge Gaozaban & Borago officinalis Linn. & Leaves & $200 \mathrm{~g}$ \\
\hline $\mathbf{4}$ & Bisfaij & Polypodium vulgare Linn. & Seeds & $75 \mathrm{~g}$ \\
\hline $\mathbf{5}$ & Sana makki & Cassia angustifolia Vahl. & Leaves & $9 \mathrm{~g}$ \\
\hline $\mathbf{6}$ & Berge Banafsha & Viola odorata Linn. & Leaves & $50 \mathrm{~g}$ \\
\hline $\mathbf{7}$ & Gule Surkh & Rosa damascena Mill. & Flower & $40 \mathrm{~g}$ \\
\hline $\mathbf{8}$ & Gule Nilofer & Nymphaea alba Linn. & Flower & $75 \mathrm{~g}$ \\
\hline $\mathbf{9}$ & Halela siyah & Terminalia chebula Retz. & Fruit & $75 \mathrm{~g}$ \\
\hline $\mathbf{1 0}$ & Tukhme Balangu & Lallemantia royleana Benth. & Seeds & $75 \mathrm{~g}$ \\
\hline $\mathbf{1 1}$ & Qand safaid & Sugar & & $4 \mathrm{~kg}$ \\
\hline
\end{tabular}

\section{METHOD OF PREPARATION OF SHARBAT AHMAD SHAHI}

SAS will be prepared as per the procedure, mentioned in (Qarabadeen-e-Majeedi) and (Bayaz-e-Kabeer Part II). First of all, the dry ingredients (mentioned above in the table) are soaked in 8 litres of water overnight and boiled next morning till only one-fourth volume of water remains. The decoction is now rubbed with the hands, cloth-filtered, and re-filtered through a cotton wool layer spread out on a sieve into another vessel. Next, the white sugar and citric acid are added to the decoction which is again heated. And any impurity that collects on the surface is removed. When the Qiwam becomes concentrated, the sodium benzoate dissolved in a little water is added, and immediately after boiling, the Qiwam is taken off the fire and sieved through a fine muslin cloth. Arq-e-gulab is now added to the Sharbat Ahmad Shahi, which is preserved in bottles.

\section{Dosage \& administration}

25-35ml with $125 \mathrm{ml}$ of Arq Gaozaban or water [6] 1-2 Tola with water [4]
Pharmacological actions [4][6]

- Cephalic tonic (Muqawwi Dimagh)

- Cardio tonic (Muqawwi Qalb)

- $\quad$ Purgative of black bile (Mushile Sauda)

Therapeutic Uses [4][6]

- Melancholia (Malikhuliya)

- Schizophrenia (Junoon)

- Mania

- Mental disorders due to excess of sauda

- $\quad$ Black bile disorders (Saudavi amraaz)

- Brain disorders (Amraze Dimaghi)

- Palpitation (Khafkan)

Pharmacological Studies

- Anxiolytic activity[179]

- Antidepressant activity[179]

- Schizophrenia[14] 
Brief description of Ingredients of Sharbat Ahmad Shahi:

Halela Siyah (Terminalia chebula Retz.)

PART USED: Fruit [24]

$>$ DOSE: $5-10 g$ [23]

> PHARMACOLOGICAL ACTIONS:

- $\quad$ Analgesic (Musakkin)[23]

- Antibilious (Dafe safra)[25]

- Astringent (Qabiz)[9]

- Blood purifier (Musaffi Khoon)[26]

- $\quad$ Brain tonic (Muqawwi Dimagh)[23]

- Cardio-tonic (Muqawwi Qalb)[24]

- $\quad$ Diuretic (Mudire Baul)[24]

- $\quad$ Eye tonic (Muqawwi Basar)[23]

- Intestinal tonic (Muqawwi Amaa)[9]

- $\quad$ Laxative (Mulaiyyan)[26]

- $\quad$ Purgative (Mushil)[25]

- $\quad$ Stomachic (Muqawwi Meda)[26]

$>$ THERAPEUTIC USES:

- Amenorrhoea (Ehtebase Tams)[24]

- Brain weakness (Zofe Dimagh)[23]

- Bronchial asthma (Zeequn Nafs)[25]

- Cardiac weakness (Zofe Qalb)[24]

- Constipation (Qabz)[25]

- Cough (Suaal)[25]

- Dementia (Zofe Hafiza)[9]

- Dysentry (Zaheer)[25]

- Dyspepsia (Tukhma)[37]

- $\quad$ Eye weakness (Zofe Basar)[23]

- Facial paralysis (Laqwa)[9]

- Fever (Humma)[26]

- Flatulence (Nafkhe Shikam)[25]

- Hepatomegaly (Azme Kabid)[26]

- Hiccup (Fuwaq)[26]

- Melancholia (Malikholia)[9]

- Piles (Bawaseer)[9]

- Rheumatism (Hudar) ([26]

- Splenomegaly (Azme Tihal)[26]

- Urinary diseases (Amraze Baul)[26]

- Vomiting (Qai)[25]

- Weakness of GIT (Zofe Meda wa Ama)[23]

- Worms[26]

\section{$>$ PHARMACOLOGICAL STUDIES}

- Anti arthritic[79]

- Anti caries activity[80]
- Anti HSV-2[71]

- Antiaging[75]

- Antibacterial [69]

- Anticonvulsant[73]

- Antidepressant[28]

- Anti-diabetic[35]

- Antifungal[76]

- Anti-inflammatory[78]

- Antimicrobial[72]

- Antioxidant[27]

- Antipyretic[77]

- Antiulcerogenic[70]

- Cardioprotective[32]

- Hepatoprotective[74]

- Immunomodulatory [31]

- Neuroprotective[33]

Bisfayej (Polypodium vulgare Linn.)

$>$ PART USED: $\operatorname{Root}[24]$

$>$ DOSE: $5-\log [7]$

$>$ PHARMACOLOGICAL ACTIONS

- $\quad$ Analgesic (Musakkin)[88]

- Antiepileptic (Mane Sara)[7]

- Anti-inflammatory (Muhallil)[8]

- Antipyretic (Dafe Humma)[88]

- Cardio tonic (Muqawwi Qalb)[7]

- $\quad$ Digestive (Hazim) [88]

- $\quad$ Diuretic (Mudire Baul)[88]

- Hypothermic[88]

- $\quad$ Laxative (Mulaiyyan)[88]

- $\quad$ Purgative of black bile \& phlegm (Mushile Sauda wa Balgham)[23]

\section{$>$ THERAPEUTIC USES}

- Asthma (Damaa)[8]

- Colitis (Qulanj)[23]

- $\quad$ Epilepsy (Sara) [8][7]

- Eruptions[7]

- Flatulence (Nafkhe shikam)[7]

- Haemorrhoids (Bawaseer)[7]

- Joint pain (Mafasil ka dard )[8]

- Leprosy (Juzam)[7]

- Melancholia (Malikhuliya)[8,7]

- Rheumatic disorders (Amraze hudar )[7]

$>$ PHARMACOLOGICAL STUDIES

- Anti-arthritic[90] 
- Anti-inflammatory[89]

- Antimicrobial[89]

- Antioxidant[89]

Aftimoon (Cuscuta reflexa Roxb.)

PART USED: Whole plant [23]

DOSE: $3-5 \mathrm{~g}$ [23]

\section{PHARMACOLOGICAL ACTIONS}

- Anodyne ([36]

- Anthelminthic (Qatile Deedan)[36]

- Anti-inflammatory (Muhallil)[24]

- Aphrodisiac (Muqawwi bah)[36]

- Carminative (Kasir riyah)[36]

- Diaphoretic[91]

- Diuretic (Mudire Baul)[23][36]

- Emmenagogue (Mudire Haiz)[25]

- Expectorant (Munaffis)[36]

- Purgative (Mushil)[91]

- Sedative (Munavim)[36]

THERAPEUTIC USES

- $\quad$ Brain disorders (Dimaghi Amraz)[7]

- Diseases of spleen (Amraze Tihal)[7]

- Epilepsy (Sara)[92]

- Facial paralysis (Laqwa)[7]

- Flatulence (Nafakhe Shikam) [91]

- Intestinal worms (Deedane Ama)[24]

- Jaundice (Yarqan)[36]

- Melancholia (Malikhuliya)[24]

- Muscles \& joint pain[36]

- Numbness (Khadar)[7]

- Palpitation (Khafqan)[7]

- Paralysis (Faalij)[7]

- Schizophrenia (Junoon)[24]

$>$ PHARMACOLOGICAL STUDIES:

- Antibacterial[95]

- Anti-fertility activity[94]

- Anticonvulsant[94]

- Cytotoxic[93]

- Anxiolytic[92]

- Anti-inflammatory[93]

Gul-e-Surkh(Rosa damacena Mill.)

PART USED: Flowers [10][11]

DOSE: $5-7 \mathrm{~g}$ [56]

> PHARMACOLOGICAL ACTIONS:
- Appetizer (Mushtahi) [57]

- Astringent (Qabiz) [58]

- Carminative (Kasire Riyah) [58]

- Refrigerant [58]

- Cardiotonic (Muqawwi Qalb) [58]

- Laxative (Mulaiyyan) ( [59]

- Expectorant(Munaffis) [57]

- Antipyretic (Dafe Humma) [59]

- Aphrodisiac (Muqawwi Bah) [59]

THERAPEUTIC USES:

- $\quad$ Sore throat (Khushunate Halq) [58]

- Tonsilitis (Warme Lauzatain) [58]

- Uterine hemorrhage [58]

- Urticaria (Shara) [58]

- Apthous ulcer [58]

- Abdominal and chest pain [60]

- Menstrual problems (Illate Haiz) [60]

- Digestive problems [60]

- Headache (Suda) [59]

- Toothache[59]

- Stomatitis (Qula)[59]

- Wound healing [59]

- Cough (Suaal) [59]

- Dementia (Zofe Hafiza) [83]

- Reflux oesophagitis [86]

> PHARMACOLOGICAL STUDIES:

- Analgesic [61][ 62]

- Antiaging [63]

- Anticonvulsant [64]

- Antibacterial [65][66][67]

- Antidepressant [69]

- Antidiabetic [70][71][72]

- Anti-HIV [73]

- Antihypertensive [74]

- Anti-inflammatory [75]

- Antioxidant [76][77][78][79]

- Antispasmodic [80]

- Antitussive [81]

Badranjboya(Nepeta hindostana Roth.)

PARTS USED: Whole plant [88][89][90][91][92][93]

DOSE: 5-7g [94][95][96]

2 Tola [97][98]

\section{> PHARMACOLOGICAL ACTIONS:}

- Exhilarent (Muffarah) [99][100][101]

- Heart tonic (Muqawwi Qalb) [96][99][100][102] 
- Concotive (Munzije sauda) [94][95][96]

- $\quad$ Gout (Dafeh Naqrus) [101]

- Astringent (Qabiz) [95][103]

- Blood purifier (Musafikhoon) [95][97][98]

- $\quad$ Purgative (Mushil) [95][104]

- $\quad$ Arthritis (Dafeh wajaul mafasil) [101][105]

- Resolvent (Muhallil) [98][99][100]

- $\quad$ Stomachic (Muqawwi Meda) [95][100][106]

- $\quad$ Brain tonic (Muqawwi Dimag) [95][107][108]

- $\quad$ Deobstruent (Muffateh Sudad) [95][109][110]

- Demulscent(Mulattif) [96][99][111]

- Calorific (Musakhin) [96][99][110]

- $\quad$ Breast inflammation (Dafeh waeme pistan) [112]

- Carminative (Kasire riyah) [94]

- $\quad$ Antipyretic (Dafeh Bukhar) [94][113]

- Antidote (Tiryaq sammom) [94]

\section{$>$ THERAPEUTIC USES:}

- Depression/Anxiety ( Parashani, Gumm) [96][99][100][104][114]

- Insomnia (Sehar) [95][104][115]

- Cough (Suaal) [98][99][100]

- Bronchitis (Warme shoeb) [95][112][104]

- Hiccup (Hichki) [100][104]

- Fever (Humma) [94][95]

- Irritable Bowel Syndrome, Colitis (Warme Qoloon) [95][99]

- $\quad$ Dyspepsia (Sue Hazam) [100][109]

- Asthma (Usre Tanafus) [95][98][99]

- Menstrual problems (Illate Haiz) [98]

- Fever (Humma) [104][112]

- Hypertension (Fisharrud dam) [94][104]

- $\quad$ Migraine (Shaqeeqa) [96]

- Influenza (common cold) [99][110][116]

- $\quad$ Shock (Gashi) [95]

- Muscular pain (Wajaul Uzlaat) [97][101][110]

- Vertigo (Duwaar) [99][109]

- Eczema (Narfarsi) [99]

- Alzheimer's disease [99][100]

- Sharpen Memory (Muqawwi Hafiza) [101][109][117]

- Antibacterial and Antifungal effects (Dafe jaraseem wa Dafe fitrasraat) [99]

- $\quad$ Leprosy (Juzaam) [95][97][112]

\section{$>$ PHARMACOLOGICAL STUDIES:}

- Anti-depressant [104]

- Anti-oxidant [118]

- Anti-spasmodic [106]

- Analgesic and Sedtion [119]
- Anti-tumor [120]

- Anti-microbial [121]

- Effect on cholinergic receptor [108]

Berge Gaozaban (Borago officinalis Linn.)

PART USED: Leaves [10]

DOSE: $5-7 \mathrm{~g}$ (Berge Gaozaban) [10] 3-5g (Gule Gaozaban) [10]

> PHARMACOLOGICAL ACTIONS:

- Cardiotonic (Muqawwi Qalb) [122][123][124]

- Brain tonic (Muqawwi Dimagh) [122][123][124]

- $\quad$ Refrigerant [122][123][124]

- Demulcent (Mulattif) [122][123][124]

- Emollient [122][123][124]

- $\quad$ Laxative (Mulaiyyan) [122][123][124]

- $\quad$ Expectorant (Munaffis) [122][123][124]

- $\quad$ Lithotriptic (Mufattite Hasat) [122][123][124]

- $\quad$ Diuretic (Mudire Baul) [125][126][127]

- Febrifuge [125][126][127]

- Antispasmodic (Dafe Tashannuj) [125][126][127]

- Diaphoretic (Moarriq) [125][126][127]

- Lactagogue [125][126][127]

- Nervine [125][126][127]

- $\quad$ Sudorific [125][126][127]

- Depurative [125][126][127]

- Anti-inflammatory (Muhallil) [125][126][127]

$>$ THERAPEUTIC USES:

- Cough (Suaal) [122][123][124]

- Coryza (Zukam) [122][123][124]

- Pneumonia (Zatur Riyah) [122][123][124]

- Pleurisy (Zatul janab) [122][123][124]

- Tuberculosis (Diq) [122][123][124]

- Asthma (Zeequn Nafas) [122][123][124]

- Meningitis (Sarsam) [122][123][124]

- Melancholia (Malikhuliya) [122][123][124]

- Functional palpitation of Heart [122][123][124]

- Jaundice (Yarqaan) [122][123][124]

- $\quad$ Stomatitis (Qula) [122][123][124]

- Kidney \& Urinary bladder stones [122][123][124]

- Cancer (Sartan) [125]

- Corns [125]

- Sclerosis [125]

- Tumours (Sala) [125]

- Bronchitis (Warme Shoeb) [125]

- Fever (Humma) [125]

- $\quad$ Sore throat (Khushunate Halq)[125]

- Swellings \& Urogenital ailments[125] 


\section{$>$ PHARMACOLOGICAL STUDIES:}

- Cardiovascular activity[13]

- Respiratory activity[13]

- Gastrointestinal activity[13]

- Antioxidant activity[180][181][182]

- Antibacterial activity[183]

Berg-e-Banafsha(Viola odarata Linn.)

PART USED: Leaves and flowers [10][11]

DOSE: Powder 3-6g [10]

Decoction 10-20ml [10]

\section{PHARMACOLOGICAL ACTIONS:}

- Anti-inflammatory (Muhallil) [127][129]

- Diaphoretic (Moarriq) [126][127]

- Diuretic (Mudire Baul) [127][129]

- Emollient [126][127]

- Expectorant (Munaffise Balgham) [127]

- Laxative (Mulaiyyan) [126][129]

- Demulcent (Mulattif) [127]

- Emetic [127]

- Purgative (Mushil) [127]

$>$ THERAPEUTIC USES:

- Cancer of Lungs, Breast or digestive tract [127]

- Whooping cough (Shaheeqa) [127]

- Headache (Suda) [127]

- Migraine (Shaqeeqa) [127]

- Insomnia (Seher) [127]

- $\quad$ Bronchitis (Warme Shoeb) [127]

- Respiratory catarrh [127]

- Asthma (Zeequn Nafas) [127]

- Mouth \& throat infections [127]

- Skin diseases [127]

\section{$>$ PHARMACOLOGICAL STUDIES:}

- Antioxidant [128]

- Analgesic [129]

- Anti-inflammatory [130]

- Antipyretic [131]

- Sedative [132]

- Hypotensive \& Lipid lowering effect [133]

- Antibacterial [134]

- Antihelmintic [135]

- Antifungal [136]

- Mosquito repellant activity [137]

\section{Sana makki (Cassia angustifolia Vahl.)}

PART USED: Leaves and root [10][11]

DOSE: $15-30 \mathrm{mg}$ [10]

500mg-2gm (Powder of leaf or pod) [10]

$>$ PHARMACOLOGICAL ACTIONS:

- Laxative (Mulaiyyan) [138]

- Ophthalmic [138]

- Liver tonic (Muqawwi Jigar) [138]

- Cardiotonic (Muqawwi Qalb) [138]

- Expectorant (Munaffise Balgham) [138]

- Febrifuge [138]

- Antiasthma [139]

- Diuretic (Mudire Baul) [139]

- Also improve the visual activity [139]

- Purgative (Mushil) [140]

$>$ THERAPEUTIC USES:

- $\quad$ Leprosy (Juzam) [141][142][143]

- $\quad$ Ringworm (Quba) [141][142][143]

- Flatulence (Nafkhe Shikam) [141][142][143]

- Colic (Qulanj) [141][142][143]

- Dyspepsia (Sue Hazm) [141][142][143]

- Constipation $(Q a b z)$ [141][142][143]

- Cough (Suaal) [141][142][143]

- Bronchitis (Warme Shoeb) [141][142][143]

- Cardiac disorders [141][142][143]

- Rheumatic disease (Amraze Hudar) [141][142][143]

- Leucoderma (Bars)[144][145]

- Eczema (Narfarsi) [144][145]

> PHARMACOLOGICAL STUDIES:

- Hypolipidemic [147]

- Antimutagenic [148]

- Hepatoprotective [150]

- Anti-inflammatory [150]

- Antishigellosis [149]

- Antibacterial [151]

- Antioxidant [155]

- Antiulcer [152]

- Antifungal [153,154]

- Antitumour [146]

- Antifertility [146]

Gul-e-Nilofer (Nymphaea alba Linn.)

PART USED: Rhizome and Flowers [11]

DOSE: Flowers-30g [8] 


\section{$>$ PHARMACOLOGICAL ACTIONS:}

- Blood purifier (Musafikhoon)) [156]

- Aphrodisiac (Muqawwi Bah) [156]

- Anodyne [156]

- Astringent (Qabiz) [156]

- Cardiotonic (Muqawwi Qalb) [156]

- Demulcent (Mulattif) [156]

\section{$>$ THRAPEUTIC USES:}

- Insomnia (Seher) [156]

- Anxiety [156]

- $\quad$ Sore throat (Khushunate Halq) [156]

- Uterine cancer [156]

- Diaphoresis [157]

- Liver disorder [158]

- Urinary disorder [158]

- Menstrual problem (Illate Haiz) [158]

- Diabetes (Ziabetus) [158]

- Dyspepsia (Sue Hazm) [167]

- Heart problem [13][50][59][68]

- Cough (Suaal) [13][50][59][68]

\section{> PHARMACOLOGICAL STUDIES:}

- Antidiabetic [159]

- Hepatoprotective [160]

- Hyperproliferative response and renal carcinogenesis [161]

- Mutagenic activity [162]

- Anti proliferative [163]

- Tumor inhibitory activity [164]

- Cholinergic activity [164]

- Analgesic [164]

- Anti-inflammatory [164]

- Antiurolithiatic [165]

- Uterotonic [166]

- Antimicrobial [164]

Tukhme Balangu (Lallemantia royleana Benth)

PART USED: Seeds [10][11]

DOSE: $5-7 \mathrm{~g}$ [10]

\section{$>$ PHARMACOLOGICAL ACTIONS:}

- $\quad$ Diuretic (Mudire Baul) [168][169]

- Tonic (Stimulant) [168][169]

- Aphrodisiac (Muqawwi Bah) [168][169]

- Antitussive [168][169]

\section{$>$ THERAPEUTIC USES:}

- Hepatic disorder [168][169]

- Renal disorder [168][169]

- Nervous disorder [168][169]

- Psychotic disease [170]

- Rheumatism (Hudar) [171]

- Joint pain [171]

- Osteoarthritis [171]

- Fever (Humma) [172]

- Common cold [172]

- Expectorant (Munaffis Balgham) [172]

- Abscesses [17]

> PHARMACOLOGICAL STUDIES:

- Antibacterial activity [173]

- Hypocholesterolemic effect [174]

- Suspending agent [175]

- Thickening agent [175]

- Anesthetic activity[176]

\section{CONCLUSION}

This review article is endeavouring to bring out that Sharbat Ahmad Shahi is one of the best Unani formulations for psychiatric disorders. It has been reported to have beneficial effect in psychiatric (mental) disorders such as depression, insanity, anxiety etc. However more scientific studies and clinical trials are needed on this compound formulation to ensure its scientific validation for clinical use in patients.

\section{REFRENCES}

[1]. Murray CJL and Lopez AD. The Global Burden of Disease: A comprehensive

Assessment of Mortality \& Disability from Disease, Injuries \& Risk factors in 1990 \&

Projected to 2020. 1996 Cambridge, MA Harvard University Press Most comprehensive

study of comparative disease burden even undertaken.Major depression estimated to be

among the most burdensome disorders worldwide.

[2]. R.C. Kessler and E.Bromet.The epidemiology of depression across cultures. Annu

Rev Public Health. Author manuscript; available in PMC 2014 July 16.

[3]. Anwar N, N.Z. Ahmed, T Shahida, K. Kabiruddin and H. Aslam. The Role of

Mufarrehat (Exhilarants) in the Management of Depression: An Evidence Based Approach. J Psychiatry 20: 420.

[4]. Kabiruddin H. Bayaze Kabir, Vol.2. Hyderabad; Hikmat Book Depot, 1938; pp. 80. 
[5]. Anonymous. National Formulary of Unani Medicine, Part I. New Delhi; Govt. Of India,

Ministry of Health \& Family Welfare, CCRUM, 2006; pp.85

[6]. Anonymous. Qarabadeen-e-Majeedi. New Delhi; All India Unani Tibbi Conference: 1986, pp.197-198.

[7]. Said HM. Hamdard Pharmacopoeia of Eastern Medicine. Delhi; Sri Satguru Publications,

A division of Indian Books Centre, 1977; pp.290.

[8]. Ghani MN, YNM. Khazainul Advia part III (Musawwer Edition). Idara Kitab-us-Shifa,

New Delhi.pp. 985-988.

[9]. Anonymous. Standardisation of single drugs of unani medicine, Part I,II, III, V. New

Delhi; CCRUM, 1992; pp. 66-72, 241-146; 13-20,148-152, 282$288 ; 79-83 ; 16-24 ; 81$ $86 ; 214-217$.

[10]. Kabirruddin, M. H., 2000. Makhzanul Mufridat Al Maroof Khawasul Advia Deoband and, Faisal Publication; pp.489,490,481,482,138,139,355,356,115,116.

[11]. Hakeem, M. A., 2011. Bustan Al Mufridat. Musawwar Edition ed. New Delhi:

Idara Kitabus-Shifa; pp.280,97,203,339,85.

[12]. Gandhipuram PSK, Palanisamy A, Durairaj SK, Sorumuthu PS Antidiabetic activity of

Fruits of Terminalia chebula on streptozoin induced diabetic rats. Journal of Health

Science, 2006; 52 (3): 283-291

[13]. Basu K. Indian Medicinal Plants, Vol. II: YNM, pp. 1741.

[14]. Tahira P, Saida H, Nudrat A, Zubairi, Waseem A, Zehra B, Sumreen B. Effect of herbal

combination on biochemical and behavioral responses in rats. Pak. J. Biochem. Mol. Biol. 2012; 45(1): 20-22

[15]. Praveen S, Prakash T, Kotresha D, Asif MA, Uday RS, Bimlesh $\mathrm{K}$, Jeevan D, Divakar

G. Antiulcerogenic activity of Terminalia chebula fruit in experimentally induced ulcer

in rats. Pharmaceutical Biology, 2011 Mar; 49(3): 262-8

[16]. Ajay K, Suja KP, Reshmi N, Aakanksha A, Nripendra NM,Satish KG. Anti-HSV-2

activity of Terminalia chebula Retz extract and its constituents, chebulagic and

chebulinic acids. BMC Complementary and Alternative Medicine, 2017 17:110.

[17]. Manoj K, Agarwal RC, Sanjay D, Rai VK, Benito J. Antimicrobial activity of aqueous extract of Terminalia chebula Retz on gram positive and gram negative

microorhanisms. International Journal of Current Pharmaceutical Research, 2009; 1(1)

[18]. Jiban D, Uday RS, Bimlesh K,Nitesh SC. Anticonvulsant activity of ethanolic extract of

fruits of Terminalia chebula on experimental animals.International Journal of Drug

Development \& Research, 2010;

[19]. Mannan A, Khan RA, Asif M. Pharmacodynamic studies on Polypodium vulgare Linn Indian J Exp Biol, 1989; 27(6): 556-60.

[20]. Ambasta SP. The Useful plants of India. New Delhi; Publications \& Information Directorate, CSIR, 1986;
[21]. Anonymous. Unani Pharmacoepia, Part I, Vol.I,II,III Vol. V. New Delhi; Department of

AYUSH, Ministry of Health \& Family Welfare, Government of India, 2007; 6, 18, 29, $32,33,106$.

[22]. Anonymous. Standardisation of single drugs of unani medicine, Part I,II, III, V. New

Delhi; CCRUM, 1992; 1, 4, 11, 20, 24, 25, 79, 98-101,268,282$283,287$.

[23]. Khare CP. Indian Medicinal Plants. New Delhi; Springer: 2007, pp. $238,365,652$ 653,654 .

[24]. Nadkarni KM. The Indian Materia Medica, vol. 1. Bombay; A.K Nadkarni Publishers: 1936, pp. 482,483, 842-843, 844-46.

[25]. Hua-Yew C, Ta-Chen L, Kuo-Hua YU,Chien-Min Y,Chun-Ching L. Antioxidant and

Free Radical Scavenging Activities of Terminalia chebula. Biol. Pharm. Bull, 2003; 26(9):1331-1335.

[26]. Kabiruddin HM. Makhzanul Mufridat Al Maroof Khawasul Advia. Deoband; Faisal Publication: 2000. pp.55, 59,79-30, 74-75, 129-30, 145-146, 19394,590-591.

[27]. Nadkarni KM. Indian Plants \& Drugs. New Delhi; Shrishti Book Distributors: YNM,pp 151,393-394, 395-397.

[28]. Chandra SR, Manohar VR, Rao SN. Antidepressant activity of aqueous extract of fruits

of Terminalia chebula in rats. International Journal of Pharmacy and Pharmaceutical

Sciences, 2012; 4(4): 449-451.

[29]. Vaibhav A, Arun KW. Immunomodulatory Activity of Alcohol Extract of Terminalia

chebula Retz Combretaceae. Tropical Journal of Pharmaceutical Research, 2011; 10(5): 567-575.

[30]. Raman S, Srinivasan BP,Kiran D.Cardioprotective effect of Terminalia chebula in type

II Diabetes induced cardiomyopathy in rat. World Journal of Pharmacy \&

Pharmaceutical Sciences, 2015; 4(10): 2151-2163.

[31]. ChiaLin C, CheSan L. Phytochemical Composition, AntioxidantActivity and

Neuroprotective Effect of Terminalia chebula Retzius Extracts. Evidence-Based

Complementary and Alternative Medicine, 2012; 1-7.

[32]. Gandhipuram PSK, Palanisamy A, Durairaj SK, Sorumuthu PS. Antidiabetic activity of

Fruits of Terminalia chebula on streptozoin induced diabetic rats. Journal of Health Science, 2006; 52(3): 283-291.

[33]. Praveen S, Prakash T, Kotresha D, Asif MA, Uday RS, Bimlesh K, Jeevan D, Divakar

G. Antiulcerogenic activity of Terminalia chebula fruit in experimentally induced ulcer In Rats. Pharmaceutical Biology, 2011; 49(3): 262-268.

[34]. Ajay K, Suja KP, Reshmi N, Aakanksha A, Nripendra NM,Satish KG. Anti-HSV-2

Activity of Terminalia chebula Retz extract and its constituents, chebulagic and

Chebulinic acids. BMC Complementary and Alternative Medicine, 2017; 17(110): 1-11.

[35]. Manoj K, Agarwal RC, Sanjay D, RAI VK, BENITO J. Antimicrobial activity of 
Aqueous Extract of Terminalia chebula Retz on gram positive and gram negative

microorhanisms. International Journal of Current Pharmaceutical Research, 2009; 1(1):

56-60.

[36]. Jiban D, Uday RS, Bimlesh K,Nitesh SC. Anticonvulsant activity of ethanolic extract of

Fruits of Terminalia chebula on experimental animals.International Journal of Drug

Development \& Research, 2010; 2(4): 764-768.

[37]. Min-Kyung C, Hyeong-Geug K, Jong-Min H,Jin-Seok L, JongSuk L, SunHo C,Chang-

GueSon. Hepatoprotective Effect of Terminalia chebula against tBHP-Induced Acute

Liver Injury in C57/BL6 Mice. EvidenceBasedComplementaryandAlternativeMedicine,

2015; 1-11.

[38]. Aranya M, Pensak J, Toshihiro A, Worapaka M, Jiradej M. In vitro anti-aging activities

of Terminalia chebula gall extract. Pharmaceutical Biology, 2010; 48(4): 469-481.

[39]. B. Rubini B, Shanthi G, Soundhari C, Rajarajan S. Antifungal activity of Terminalia

chebula and Terminalia catappa on two dermatophytes. Journal of Medicinal and

Aromatic Plants, 2013; 4(2): 15-19.

[40]. Rajyalakshmi N, Nagesh RG, Ramanath B. Evaluation of local anaesthetic and

antipyretic activity of Terminali chebula Retz in experimental animal models

International Journal of Pharma and Bio Sciences, 2015; 6(1): 219-225

[41]. Safkath IJ, Zakia S, Ershad A, Marium B, Mominul H. Evaluation of Analgesic and

Anti-Inflammatory Activities on Ethanolic Extract of Terminalia chebula Fruits in

Experimental Animal Models.American Journal of Plant Sciences, 2014; 63-69.

[42]. Nichole M, Ramesh GC, Natalia V, Katie K, Jean M, John GT, Michelle LA, Terry

CD,Sanyasi RK. Evaluation of Terminalia chebula Extract for Anti-Arthritic Efficacy

And Safety in Osteoarthritic Dog. Veterinary Science \& Technology, 2016; 7(1): 1-8.

[43]. Carounanidy U, Satyanarayanan R, Velmurugan A. Use of an aqueous extract of

Terminalia chebula as an anticaries agent: A clinical study. Indian J Dent Res, 2007;

18(4): 152-155.

[44]. Veena S, Manu S.Operculina turpethum as a panoramic herbal medicine: A Review.

International Journal of Pharmaceutical Sciences and Research, 2012; 3(1): 21-25.

[45]. Ghani N. Khazainul Advia. Delhi; Idara Kitabus Shifa, YNM, pp. $187-89,493,526,792$, 793.

[46]. Hakeem MA. Bustanul Mufradat. New Delhi; Idara Kitabus Shifa, 2002; 60, 67-68, 72, 85-86, 90-91, 92,347,348.

[47]. Gaamoune S, Nouioua W, Djaout O. Antioxidant, antimicrobial and anti-inflammatory activities of flavonoids and tannins extracted from Polypodium vulgare L. Asian Journal of Biochemical and Pharmaceutical Research, 2015; 5(4): 114 122.

[48]. Mannan A, Khan RA, Asif M. Pharmacodynamic studies on Polypodium vulgare Linn. Indian J Exp Biol, 1989; 27(6): 556-60.

[49]. Ambasta SP. The Useful plants of India. New Delhi; Publications \& Information

Directorate,CSIR, 1986; 152.

[50]. Basu K. Indian Medicinal Plants, Vol. II: YNM, pp. 1741.

[51]. Sujith T, Sapna S, Velmurugan C, Ashok BS. Evaluation of anxioltic activity of whole

plant of Cuscuta reflexa.World Journal of Pharmacy and Pharmaceutical Sciences, 2015; 4(8): 1245-1253.

[52]. Pavan BU, Suggala VS, Chandrashekhar DU. Preliminary screening of Cuscuta reflexa stems for Anti inflammatory and cytotoxic activity. Asian Afrin et al. European Journal

of Biomedical and Pharmaceutical Sciences www.ejbps.com 206 Pacific Journal of

Tropical Biomedicine, 2012; 1303-1307.

[53].Malaya G, Upal KM,Dilipkumar P, Shiladitya B, Sumit C. Studies on brain biogenic

amines in methanolic extract of Cuscuta reflexa Roxb and Corchorus olitorius Linn seed

treated mice. Acta Poloniae Pharmaceutica-Drug Reasearch, 2003; 60(3): 207-210.

[54].Wiart C. Ethnopharmacology of Medicinal Plants. Asia and the Pacific Humana Press,

2006; 143.

[55]. IbnSina. Al Qanoon fil Tib (Urdu Translation by Kantoori GH) Vols. 1, part 2, part

3, New Delhi;Idara Kitabus Shifa, 2007, pp. 39-40,57,61,8384,212,82-92.

[56]. Lubhaya R. Goswami bayanul advia. Vol. I. Goswami Pharmacy, Qasim Jan Street, Delhi;1982. p. 295-297.

[57]. Chatterjee A, Pakrashi SC. The treatise on Indian medicinal plants.Vol. II. Publication \& Information Directorate, CSIR, NewDelhi;1992. p. 15-16.

[58]. NadkarniAk. Indian Material Medica. Vol. I. Bombay Popular Prakashan, Mumbai; 1954. p. $1072-1073$.

[59]. Kirtikar KR, Basu BD. Indian Medicinal Plants. Vol. II. Connaught Place, Dehradun; 1991.p. 1072-1073.

[60]. Boskabady MH, Shafei MN, Saberi Z, Amini S. Pharmacological effects of Rosa

damascena. Iranian Journal of Basic Medical Sciences. 2011;14(4):295-307.

[61]. Rakhshandeh H, Vahdati-Mashhadian N, Dolati K, Hosseini M. Antinoceptive effect of

Rosa damascena in mice. Journal of Biological Sciences. 2008; 8(1):176-180.

[62]. Hajhashemi V, Ghannadi A, Hajiloo M. Analgesic and antiinflammatory effects of

Rosa damascena hydro alcoholic extract and its essential oil in animal models. Iranian Journal of Pharmaceutical Research. 2010; 9(2):163-168.

[63]. Jafari M, Zarban A, Pham S, Wang T. Rosa damascena decreased mortality in adult Drosophila. Journal of Medicinal Food.2008;11(1):9-13.

[64]. Kheirabadi M, Moghimi A, Rakhshande H, Rassouli MB. Evaluation of the 
anticonvulsant activities of Rosa damascena on the PTZ induced seizures in wistar rats.

Journal of Biological Sciences.2008; 8:426-430.

[65]. Kurhade BB, Vite MH, Nangude SL. Antibacterial activity of Rosa damascene Miller.

International Journal of Research in Pharmaceutical and Biomedical Sciences.

2011; 2(3):1015-1020

[66].Ulusoy S, Boşgelmez-Tinaz G, Seçilmiş-Canbay H. Tocopherol, carotene, phenolic

contents and antibacterial properties of rose essential oil, hydrosol and absolute. Current

Microbiology. 2009; 59(5):554 -558.

[67]. Halawani EM. Antimicrobial activity of Rosa damascena petals extracts and chemical

composition by gas chromatography-mass spectrometry (GC/MS) analysis. African

Journal of Microbiology Research. 2014; 8(24):2359-2367.

[68]. K.R. Kritikar \& B.D. Basu, Indian Medicinal Plants, International Book Distributors

Dehradun 2005, vol 1, P.205-206.

[69]. Dolati K, Rakhshandeh H, Shafei MN. Antidepressant-like effect of aqueous extract

from Rosa damascena in mice. Avicenna Journal of Phytomedicine. 2011; 1(2):91-97.

[70]. Ju JE, Joo YH, Chung N, Chung SY, Han SH, Lee YK. Antidiabetic effects of red rose

flowers in streptozotocin-induced diabetic mice.Journal of Korean Society for Applied

Biological Chemistry.2014; 57(4):445-448.

[71]. Gholamhoseinian A, Fallah H, Sharifi-far F. Inhibitory effect of methanol extract of

Rosa damascena Mill. flowers on alphaglucosidase activity and postprandial

hyperglycemia in normal and diabetic rats. International Journal of Phytotherapy and

Phytopharmacology.2009;16(10):935-941.

[72]. Gholamhoseinian A, Fallah H, sharifi-far F, Mirtajaddini M. The inhibitory effect of

some Iranian plants extracts on the alpha glucosidase. Iranian Journal of Basic Medical

Sciences. 2008; 11(1):1-9.

[73]. Mahmood N, Piacente S, Pizza C, Burke A, Khan AI, Hay AJ. The anti-HIV activity

And mechanisms of action of pure com-pounds isolated from Rosa

damascena.Biochemical and Biophysical Research Communication. 1996;229(1):73-79.

[74]. Kwon EK, Lee DY, Lee H, Kim DO, Baek NI, Kim YE, Kim HY. Flavonoids from the

buds of Rosa damascene inhibit the activity of 3-hydroxy-3methylglutaryl-coenzyme a

reductase and angiotensin I-converting enzyme. Journal of Agricultural and Food

Chemistry. 2010; 58(2):882-886.

[75]. Maleev A, Neshtev G, Stoianov S, Sheikov N. The ulcer protective and antiinflamatory

effect of Bulgarian rose oil. Eksperimental na Meditsinai Morfologiia.1972; 11(2):55-

60.

[76]. Kumar N, Bhandari P, Singh B, Bari SS. Antioxidant activity and ultra-performance

LC- electrospray ionization-quadrupole timeof-flight mass spectrometry for phenolics-

based fingerprinting of rose species: Rosa damascena, Rosa bourboniana and Rosa
brunonii.Food and Chemical Toxicology.2009; 47(2):361-367.

[77].Ozkan G, Sagdiç O, Baydar NG, Baydar H. Antioxidant and antibacterial activities of

Rosa damascena flower extracts. Food Science and Technology International.

2004; 10(4):277-281.

[78]. Yassa N, Masoomi F, Rohani Rankouhi SE, Hadjiakhoondi A. Chemical composition

And antioxidant activity of the extract and essential oil of Rosa damascena from Iran,

population of Guilan. DARU Journal of Pharmaceutical Sciences. 2009; 17(3):175-180

[79]. Shahriari S, Yasa N, Mohammadirad A, Khorasani R, Abdollahi M.In vivo antioxidant potential of Rosa damascena extract from Guilan, Iran comparable to $\alpha$-tocopherol.

International Journal of Pharmacology. 2007; 3(2):187-190.

[80]. Bani S, Hasanpour S, Mousavi Z, Garehbaghi PM, Gojazadeh M. The effect of Rosa

damascena extract on primary dysmenorrhea: a double-blind cross-over clinical trial.

Iranian Red Crescent Medical Journal. 2014; 16(1):1-6.

[81]. Shafei NM, Rakhshandah H, Boskabady HM. Antitussive effect of Rosa damascena in

Guinea pig. Iranian Journal of Pharmaceutical Research. 2003 ;(2): 231-234.

[82]. Boskabady MH, Vatanprast A, Parsaee H, Ghasemzadeh M. Effect of aqueous-ethanolic

extract from Rosa damascena on guinea pig isolated heart. Iranian Journal of Basic

Medical Sciences. 2011; 14(2):116-121.

[83]. Awale S, Tohda C, Tezuka Y, Miyazaki M, Kadota S. Protective effects of Rosa

damascena and its active constituent on $\mathrm{A} \beta$ (25-35)-induced neuritic atrophy. Evidence

Based Complementary and Alternative Medicine. 2011; (2011); Article ID 131042: 1-8.

[84]. Rakshanda H, Hosseini M. Potentiation of phenobarbital hypnosis by Rosa damascene in mice. Indian Journal of Experimental Biology. 2006; 44(11):910-912.

[85]. Arezoomandan R, Kazerani HR, Behnam-Rasooli M. The laxative and prokinetic

effects of Rosa damascenaMill in rats. Iranian Journal of Basic Medical Sciences. 2011;

14(1): 9-16.

[86]. Sengottuvelu S, Srinivasan D, Ramasamy S. The Effect of polyherbal formulation-PHF

on experimentally induced reflux esophagitis in rats. Journal of Pharmacy Research.

2008; 1(1):11-15.

[87]. Haleem, A., MufradateAzizi (Urdu), MatbuaSahityaMandir Press Ltd., Lucknow 1948, p. 83,86 .

[88]. Majoosi, A. Kamilus Sanaah (Urdu Translation by Ghulam Husain Kantoori), Matba

Nami Munshi Naval Kishore1889; V. 2, p. 313

[89]. Bolkent S, Yanardag R, Karabulut-Bulan O, et al. Protective role of Melissa officinalis

L.extract on liver of hyperlipidemic rats: a morphological and biochemical study.

Journal of ethnopharmacology 2005; 99:391-398

[90]. Ibn Baitar. Al Jami ul Mufradatul Adviawal Aghzia. (Urdu translation CCRUM). New

Delhi: Ministry of Health and Family Welfare, Govt. of India1999; Vol. 3rd p.185. 
[91]. Ibn Kitabul Qanoon-fit-Tib (Translation), Matba Nawal Kishore, Lucknow, Edition I1037 H; p. 57.

[92]. Kabeeruddin, H., (1924) KulliyatAdvia(Urdu), IdaraTameerTibb, Urdu Bazar, Lahore, p. 856-954.

[93]. Samarqandi, N., MualijateSharheAsbab(Urdu translated by Kabeeruddin), Hikmat Book

Depot, Hyderabad1916; V. 1, p. 156.

[94]. Lubhaya, R. H., (NA) Goswami Bayanul Advia, Goswami Kutub Khana Gali Qasim

Jaan, Delhi, V. 1, p. 122.

[95]. Ibn Baitar Aljamiul Mufradatal Adviawal Aghzia (Urdu translation), Central Council for

Research in Unani Medicine, Ministry for Health and Family Welfare, New Delhi1985,

V. 1, p. 177-179.

[96]. Kabiruddin, H., () BayazeKabir(Urdu), Hikmat Book Depot, Hyderabad1935; pp.

$313,218,189$.

[97]. Nadkarni K. M., Indian Materia Medica, Bombay Popular Prakashan, Bombay, India

1976; V.1, pp. 786.

[98]. Hakim, M. A., (BustanulMjufradat(Urdu), KarkhanaJamitulAdvia, Lucknow, India1893; pp.83, 188.

[99]. Haleem, A., MufradateAzizi (Urdu), MatbuaSahityaMandir Press Ltd., Lucknow 1948, pp.83, 86.

[100]. Majoosi, A. Kamilus Sanaah (Urdu Translation by Ghulam Husain Kantoori), Matba

Nami Munshi Naval Kishore1889; V. 2, pp. 313.

[111]. Kabeeruddin, H., (1924) KulliyatAdvia(Urdu), IdaraTameerTibb, Urdu Bazar, Lahore, pp. 856-954.

[112]. Samarqandi, N., MualijateSharheAsbab(Urdu translated by Kabeeruddin), Hikmat

Book Depot, Hyderabad1916; V. 1, pp. 156

[113]. Holmes JM. Thomas Jefferson treats himself: herbs, physicke, \& nutrition in early

America: Loft Press, Incorporated, 1997

[114]. Perry N, Perry E. Aromatherapy in the management of psychiatric disorders: clinical

And neuropharmacological perspectives. CNS drugs 2006; 20:257-280

[115]. Bolkent S, Yanardag R, Karabulut-Bulan O, et al. Protective role of Melissa officinalis

L.extract on liver of hyperlipidemic rats: a morphological and biochemical study.

Journal of ethnopharmacology 2005; 99:391-398

[116]. Kitano, Y., Usui, C., Takasuna, K., Hirohashi, M., Nomura, M., Increasing-current

electroshock seizure test. A new method for assessment of antiand proconvulsant

activities of drugs in mice, J PharmacoloToxicol methods1996; V. 35, pp. 25-29.

[117]. Gupta VK, Sharma SK. Plants as natural antioxidants. Nat. Prod. $\operatorname{Rad} 2006 ; 5: 326-$ 334.

[118]. Mimica-Dukic N, Bozin B, Sokovic M, et al. Antimicrobial and antioxidant activities

of Melissa officinalis L.(Lamiaceae) essential oil. Journal of agricultural and food

chemistry 2004; 52:2485-2489

[119]. Plat for a future, [ http://www.pfaf.org/User/plant. aspx?LatinName $=$ Viola+odorata] cited- 2017March 9.

[120]. Dudai N, Weinstein Y, Krup M, et al. Citral is a new inducer of caspase-3 in tumor

cell lines. Planta Medica-Natural Products and Medicinal Plant Research 2005;

71:484-487.

[121]. Allahverdiyev A, Duran N, Ozguven M, et al. Antiviral activity of the volatile oils of

Melissa officinalis L. against Herpes simplex virus type-2. Phytomedicine 2004;

11:657-661.

[122]. Ghani N, Khazain ul advia, Vol 6.

[123]. Hasan Masoda et al, 1982. Jour Chem Soc Pak., 4(4): 281-283.

[124]. Tewari NC et al, 1970. Indian Jour Chem, 8(7): 593-597.

[125]. A handbook of Medicinal Herbs. James A Duke.

[126]. Green Medicine. Leyel C.F, 93.

[127]. Thoronton J, 1810. New family of Herbal Medicine., 167.

[128]. Ebrahimzadeh MA, Nabavi SM, Nabavi SF, Bahramian F, Bekhradnia AR.

Antioxidant and free radical scavenging activity of $H$. officinalis L. var. angustifolius,

V. odorata, B. hyrcana and C. speciosum. Pak J Pharma Sci. 2010; 23, 29-34.

[129]. Barkatullah, Ibrar M, Ali N, Muhammad N, Meryam E. In vitro pharmacological study

and preliminary phytochemical profile of Viola canescens Wall. Ex Roxb. Afri J Pharm

Pharmacol. 2012; 6: 1142-46.

[130]. Koochek MH, Pipelzadeh MH, Mardani H. The effectiveness of Viola odorata in the

prevention and treatment of formalininduced lung damage in the rat. J Herbs Spices

Med Plants.2003; 10: 95-103.

[131]. Khattak SG, Gilani SN, Ikram M. Antipyretic studies on some indigenous Pakistani medicinal plants. J Ethnopharmacol.1985; 14: 45-51.

[132]. Alireza M, Ali R. Evaluation of sedative and preanestheticeffects of Viola odorata

Linn. extract compared with diazepam in rats. Bull. Env. Pharmacol. Life Sci. 2013; 2: 125-31.

[133]. Siddiqi HS, Mehmood MH, Rehman NU, Gilani AH. Studies on the antihypertensive

and antidyslipidemic activities of Viola odorata leaves extract. Lipids Health Dis.

2012;11: 6.

[134]. Ramezani M, Zarrinkamar F, Bagheri M, Rajabnia, R. Study of environment

temperature effect on the antibacterial activity of water extract of different organs of

Viola odorata in the different stages of growth. J Babol Univ Med Sci. 2012; $14: 16$ -21 .

[135]. Colgrave ML, Kotze AC, Ireland DC, Wang CK, Craik DJ. The anthelmintic activity

Of the cyclotides: Natural variants with enhanced activity. Chem Bio Chem, 2008, 9: 1939-45.

[136]. Pawar V, Thaker V. In vitro efficacy of 75 essential oils against Aspergillus niger. Mycoses 2006; 49: 316-23.

[137]. Amer A, Mehlhorn H. Repellency effect of forty-one essential oils against aedes, anopheles and culex mosquitoes. Parasitol Res. 2006; 99: 47890. 
[138]. Hu S. Food Plants of China. Chinese University Press; 2005. p. $1-844$.

[139]. Chauhan NS. Medicinal and aromatic plants of Himachal Pradesh. Indus Pub. Co. New Delhi. 1999; 151-52)

[140]. Manojlovic I, Bogdanovic-Dusanovic G,Gritsanapan W, Manojlovic N. Isolation and

Identification of anthraquinones of Caloplaca cerina and Cassia species. Chemical Pap 2006; 60(6):466-68.

[141]. Hooker, J.D., the Flora of British India, Vol.II, L.Reeve and Co., England, 1879 ,

pp.26. Kirtikar, K.R. and Basu, B.D., Indian Medicinal Plants, Vol II, Periodical

Experts D- 42, Vivek Vihar Delhi, 1975, pp.877.

[142]. Jain, S.K., Medicinal Plants, National Book Trust, New Delhi., 1968, pp.37.

[143]. Maitya TK, Mandal SC, Mukherjee PK. Saha K, Dass J, Saha BP, et al Evaluation of

hepatoprotective potential of Cassia species leaf extract, Nat. Prod. Sci 1997; 3:122.

[144]. Chauhan NS. Medicinal and aromatic plants of Himachal Pradesh. Indus Pub. Co. New Delhi. 1999;151-52.

[145]. Anonymous. The Wealth of India A dictionary of Indian raw materials \&

Industrial products, Publications \& Information Directorate, C.S.I.R., Vol.3, pp. 368-70.

[146]. Anushia.C, Sampathkumar.P and Ramkumar.L. Global Journal ofPharmacology. 3 (3):127-130, 2009)

[147]. Tiwari P, Kumar K, Panik R., Hepatoprotective effects of Cassia species

whole plant. International Journal of Pharmacy \& Technology $2011 ; 3(2): 2798$

2806.

[148]. Patil UK, Saraf S, Dixit VK. Hypolipidemic activity of seeds of Cassia species Linn. J. of Ethnopharmacol 2004;90 (2-3):249-252.

[149]. Choi JS, Lee HJ, Park KY, Ha JO, Kang SS. In vitro antimutagenic effects of anthraquinone aglycones and naphthopyrone glycosides from Cassia species. Planta Med 1997; 63 (1):11-14.

[150]. Maitya TK, Mandal SC, Mukherjee PK. Saha K, Dass J, Saha $\mathrm{BP}$, et al Evaluation of hepatoprotective potential of Cassia species leaf extract, Nat. Prod. Sci 1997; 3:122.

[151]. Singh VK, Khan A M. Medicinal Plants and Folklores - A Stratergy towards

Conquest of Human Ailments. Vol. 9.Today \& Tomorrow Printers \& Publishers, 1990:67.

[152]. Gill NS, Sharma A, Arora R, Bali M. Evaluation of Cassia species seeds for their antioxidant and antiulcer activity. J Med Sci 2011; 11 (2):96-101.

[153]. Mukharjee PK, Saha K, Das J, Saha BP,Pal M. Antifungal activity of the leaf extract

of Cassia species linn, Phytother. Res. 1996; 10:521-522.

[154]. Acharya TK, Chatterjee IB, Isolation of chrysophanic acid -9anthrone, a fungicidal compound from Cassia species, Sci.Cult.1974; 40:376.

[155]. Chakrabarty K. Chawla H. Terpenoids and phenolics from Cassia species stem bark",
Indian J. Chem. 1983;22(B): 1165-1166.

[156].

http://www.pfaf.org/user/Plant.aspx?LatinName=Nymphaea + alba

[157]. Aspects of Asian medicine and its practice in the west by W.C Evans in Trease and

Evans Pharmacognosy $15^{\text {th }}$ edition W.B Saunders publications 2002, pp.no 477 .

[158]. Mohan Maruga Raja, M. K., Sethiya, Neeraj Kumar and Mishra S. H. (2010). A

comprehensive review on Nymphaea stellata: A traditionally used bitter. J Adv Pharm

Technol Res., 010 Jul-Sep; 1(3): 311-319.

[159]. Rajagopal, S. and Sasikala, k. 2008. Antidiabetic activity of hydro-ethanolic extracts of

Nymphaea stellata flowers in normal and alloxan induced diabetes rats. Afri. $J$.

Pharm. Pharmacol. 2: 173-178.

[160]. Bhandarkar, M.R. and Khan, A. 2004. Antihepatotoxic effect of Nymphaea stellata

Willd. against carbon tetra chloride induced hepatic damage in albino rats. $J$.

Ethnopharmacol. 91: 61-64.

[161]. Khan, N. and Sultana, S. 2005. Chemomodulatory effect of Ficus racemosa extract

against chemically induced renal carcinogenesis and oxidative damage response in

Wistar rats. Life Sci. 29: 1194-1210.

[162]. Sowemimo, A.A., Fakoya, F.A., Awopetu, I.,Omobuwajo, O.R. and Adesanya, S.A.

2007. Toxicity and mutagenic activity of some selected Nigerian plants.

J. Ethnopharmacol. 113: 427-432.

[163]. Selvakumari, E., Shantha, S., Puroshothprabhu, T. And Sreenathkumar, C. 2012.

Antiproliferative activity of Ethanolic flower extract from Nymphaea pubescens willd

Youth Education and Research Trust (YERT) jairjp.com Selvakumari et al., 2016

against human cervical and breast carcinoma in vitro.Int. Res. J. Pharm. 3: 124-125.

[164]. Raja, M.M., Sethiya, N.K. and Mishra, S.H. 2010. A comprehensive review on

Nymphaea stellata: A traditionally used bitter. J. Adv. Pharmaceut. Technol. Res. 3: 311-319.

[165]. Bhaskar, V.H., Shelke, S. and Tushar, T. 2012. Effect of ethanolic extract of

Nymphaea alba Linn on urolithiatic rats. Int. J. Pharm. Pharmaceut. Sci. 4: 572-574.

[166]. Anindya, B., Moumita, S., Sudhanshu, S.R. and Sudam, C.S. 2014. Uterotonic

properties of Nymphaea alba on Youth Education and Research Trust (YERT)

jairjp.com Selvakumari et al., 2016 isolated myometrium model. Int. J. Pharm.

Pharmaceut. Sci. 6(6): 490-493.

[167]. Sowemimo, A.A., Fakoya, F.A., Awopetu, I., Omobuwajo, O.R. and Adesanya, S.A.

2007. Toxicity and mutagenic activity of some selected Nigerian plants. $J$.

Ethnopharmacol. 113: 427-432.

[168]. Amin GR. 2005. Popular Medicinal Plants of Iran. Publications of Tehran University

Of Medical Sciences, Tehran. 66-67.

[169]. Ghannadi A, Zolfaghari B. 2003. Compositional analysis of the essential oil of 
Lallemantia royleana (Benth. In Wall.) Benth. From Iran. Flavour Frag J, 18: 237-239.

[170]. Safa O, Soltanipoor MA, Rastegar S, Kazemi M, Nourbakhsh Dehkordi Kh, Ghannadi

A. 2013. An ethnobotanical survey on hormozgan province, Iran. Avicenna J

Phytomed,3: 64-81

[171]. Moghaddam TM, Razavi SMA, Emadzadeh B. Rheological interactions between

Lallemantia royleana seed extract and selected food hydrocolloids. J. Sci. Food Agri, 2011; 91: 1083-1088.

[172]. Naghibi F. Labiatae family in folk medicine in Iran. Iranian J. Pharm. Sci., 2005; (1): 200-210

[173]. Karcioglu L, Tanis H, Comlekcioglu N, Diraz E, Kirecci E, Aygan A (2011)

Antimicrobial Activity of Salvia trichoclada in Southern Turkey. Int. J. Agric. Biol.

13:134-136.

[174]. Kanakavalli K, Thillaivanan S, Parthiban P, Vijayalakshmi G, Sudha M, Sutha J. 2014.

Anti-hyperlipidemic herbs in siddha system of medicine. Int $\mathbf{J}$ Pharma Sci, 4: 541-545.

[175]. Shanmygam S, Manavalan R, Venkappayya D. Natural polymer and their application.

Natural product radiance, 2005; 4(6): 478-481

[176]. Rabi Atabaki and Majid Hassanpour-ezatti, Improvement of Lidocaine Local

Anesthetic Action Using Lallemantia royleana Seed Mucilage as an Excipient. Iranian

Journal of Pharmaceutical Research (2014), 13 (4): 1431-1436.

[177]. Kabeeruddin M. Bayaz kabeer Part 3. New Delhi: Idara Kitabush Shifa pp 71-77, 2010.

[178]. National formulary of Unani Medicine, Part -VI. Govt. of India, Ministry of Health \& Family Welfare, CCRUM; 2011: 116.

[179]. Muhammad A, Aisha A. Decreased brain serotonin turnover rate following

administration of Sharbat-e-Ahmed Shah produces antidepressant and effect in rats.

Springer Science+Business Media, LLC 2017, Metab Brain Dis (2017) 32:1785-1790.

[180]. B. Mhamdi, W. Aidi Wannes, J. Sriti, I. Jellali, R. Ksouri, B. Marzouk, "Effect of

harvesting time on phenolic compounds and antiradical scavenging activity of Borago

officinalis seed extracts",Industrial Crops and Products, 2010, Vol. 31(1); 1-4.

[181]. Mikel García-Iñiguez de Ciriano, Cecilia García-Herreros, Eduardo Larequi, Idoia

Valencia, DianaAnsorena, Iciar Astiasarán, "Use of natural antioxidants from

lyophilized water extracts of Borago officinalis in dry fermented sausages

enriched in $\omega$-3 PUFA",Meat Science, 2009,Volume 83(2); 271277.

[182]. Mahinda Wettasinghe, Fereidoon Shahidi,“Antioxidant and free radical-scavenging properties of ethanolic extracts of defatted borage (Borago officinalis L.) seeds", Food Chemistry, 1999, Vol.67(4); 399-414.

[183]. CEYLAN, E. and FUNG,D.Y.C. 2004. Antimicrobial activity of spices.

J.RapidMeth.Aut.Mic. 12, 1-55.

\section{AUTHORS PROFILE}

Afshan khan presently pursuing M.D in Pharmacology at Jamia Hamdard University New Delhi, India. Mrs Aisha Siddiqui is currently working as Assistant Professor in Department of Ilmul Avia at Jamia Hamdard University New Delhi, India. Mr M. A Jafri is currently working as Professor in Department of Ilmul Advia at Jamia Hamdard University New Delhi, India and Mr Mohd Asif is currently working as Assitant Professor in Department of Ilmul Advia at Jamia Hamdard University New Delhi, India. 